\title{
A Low Order Model of SCR-in-DPF Systems with Proper Orthogonal Decomposition
}

Seun Olowojebutu and Thomas Steffen Loughborough University

Citation: Olowojebutu, S. and Steffen, T., "A Low Order Model of SCR-in-DPF Systems with Proper Orthogonal Decomposition,"

SAE Technical Paper 2018-01-0953, 2018, doi:10.4271/2018-01-0953.

\section{Abstract}

his paper presents a method to achieve a low order system model of the urea-based SCR catalyst coated filter (SCR-in-DPF or SCRF or SDPF), while preserving a high degree of fidelity. Proper orthogonal decomposition (POD), also known as principal component analysis (PCA), or Karhunen-Loéve decomposition (KLD), is a statistical method which achieves model order reduction by extracting the dominant characteristic modes of the system and devises a low-dimensional approximation on that basis. The motivation for using the POD approach is that the low-order model directly derives from the high-fidelity model (or experimental data) thereby retains the physics of the system. POD, with
Galerkin projection, is applied to the $1 \mathrm{D}+1 \mathrm{D}$ SCR-in-DPF model using ammonia surface coverage and wall temperature as the dominant system states to achieve model order reduction. The performance of the low-order POD model (with only a few basis modes) shows good agreement with the high fidelity model in steady and transient states analyses. This shows the promise of the application of POD in exhaust aftertreatment system (EATS) modelling to achieve high fidelity low order models. In addition system control design is easier for the reduced order model using a modern approach. We demonstrate the performance of a model-based controller applied to the low-order POD model to minimize ammonia slip for a transient test cycle.

\section{Introduction}

engines offer superior performance in fuel economy compared to gasoline engines [1], but the simultaneous control of soot / particulates (PM) and nitrogen oxides $\left(\mathrm{NO}_{\mathrm{x}}\right)$ is challenging. In modern diesel engines, exhaust after-treatment systems (EATS) are required to meet the emission standards for $\mathrm{PM}$ and $\mathrm{NO}_{\mathrm{x}}$. A typical diesel EATS comprise diesel oxidation catalyst (DOC), diesel particulate filter (DPF), urea-based selective catalytic reduction (SCR) and ammonia oxidation (AMOX) units. Integration of the DPF and the urea-SCR catalyst in a single block (SCR-in-DPF or SCRF or SDPF) is an emerging technology to meet more stringent future emission standards $[\underline{2}, \underline{3}]$. This is expected to save cost (as total package weight and volume is reduced), and offer improved cold start performance (as thermal mass is lower).

Modelling of the SCR-in-DPF system is complicated. The main challenge is how to best capture the complexity of the physical and chemical phenomena-e.g., the competition of soot oxidation and SCR reaction for available $\mathrm{NO}_{2}$, or the interaction of washcoat loading on deNOx performance, $\mathrm{PM}$ filtration efficiency and system pressure drop-in a simplified but adequate representation [4]. Therefore research is ongoing on the development of an SCR-in-DPF model which achieves the right balance between adequacy and complexity, and which can form the basis of a control algorithm that can be implemented within an engine control unit (ECU).
Proper orthogonal decomposition (POD) can be used for model order reduction in EATS modelling. The POD method is a multi-variate statistical method that aims at obtaining a compact representation of data. In the POD method, large scale system data is decomposed into its characteristic modes (or eigenvectors). A Galerkin projection of the large scale data on the subspace spanned by the largest (or dominant) eigenvectors can be used to derive a lower dimensional surrogate of the original large scale system.

In the POD method, system data obtained from numerical simulation or experiments is decomposed into a linear combination of basis functions (POD modes or eigenvectors) and associated coefficients. The POD modes are obtained from a singular value decomposition of the system data. The full order model is then approximated by the largest POD modes with Galerkin projection. The motivation for using the POD approach is that the low-order model directly derives from the high-fidelity model (or experimental data) thereby retains the physics of the system.

POD has been applied to engine research to study turbulence and cyclic variation of flow and combustion properties in internal combustion engines $[\underline{5}, \underline{6}]$. The method is used extensively in model reduction in fluid dynamics problems [7].The Galerkin technique has been applied in EATS modelling in the context of mean weighted residuals method of solution of differential equations $[\underline{8}, \underline{9}]$. In $[\underline{8}, \underline{9}]$, continuous piecewise trial functions are used to approximate the 
time-dependent PDEs. The POD-Galerkin technique extends this method with the POD modes used as the trial functions for model approximation. Since the POD modes are obtained from system data, this approach leads to a better approximation of the underlying model. The authors are unaware of previous application of the POD method to EATS model order reduction.

In this paper POD is applied to the development of a low order 1D + 1D SCR-in-DPF model. We demonstrate the application of the POD method for SCR-in-DPF model order reduction. We apply POD to our 1D + 1D SCR-in-DPF model using ammonia surface coverage and wall temperature as system states. The "full order" SCR-in-DPF model contains equations for continuity, momentum and energy conservation. Soot accumulation dynamics is not included in the model at this stage, and the full order model is to be validated. However the reduced order POD model with only a few POD modes performed well in approximating the full order model in the simulated steady state, transient state and control system applications. This demonstrates the conceptual validity of the approach.

At this stage, soot dynamics is not considered in our model. As such the conclusions we present here are tentative. Overall system dynamics will change with soot accumulation and it will be interesting to see how the POD approach performs in that context. Our ongoing work will address this. This paper is prepared to introduce the POD approach in EATS modelling following initial results which look promising.

The rest for the paper is organised as follows. An outline of the 1D + 1D SCR-in-DPF model is first presented. Thereafter the POD method is introduced. Some background on the mathematical formulation of the POD method is presented. This is followed by its application to SCR-in-DPF modelling. The performance of the reduced order (POD) model is then presented for steady state, transient state and control applications, followed by some concluding remarks.

\section{D+1D SCR-in-DPF Model}

\section{Model Formulation}

The catalysed DPF model is adapted for SCR in DPF modelling [10]. The model comprises continuity, momentum and energy conservation equations for the representative inlet and outlet channels in the axial direction and the transverse flow through the filter wall (Figure 1). Ammonia surface coverage on the filter wall is considered, but soot deposition and oxidation dynamics is not included in this work. The assumptions taken for the $1 \mathrm{D}+1 \mathrm{D}$ catalysed DPF model development can be found in ref [11].

An outline of the $1 \mathrm{D}+1 \mathrm{D}$ SCR-in-DPF model $[\underline{12}, \underline{13}, \underline{14}]$ is presented with additional comments on the particular considerations taken in this work. As per Figure $1, z$ is the axial dimension along the catalyst length, and $x$ is the dimension perpendicular to the wall surface.

\section{FIGURE 1 Schematic of a filter channel}

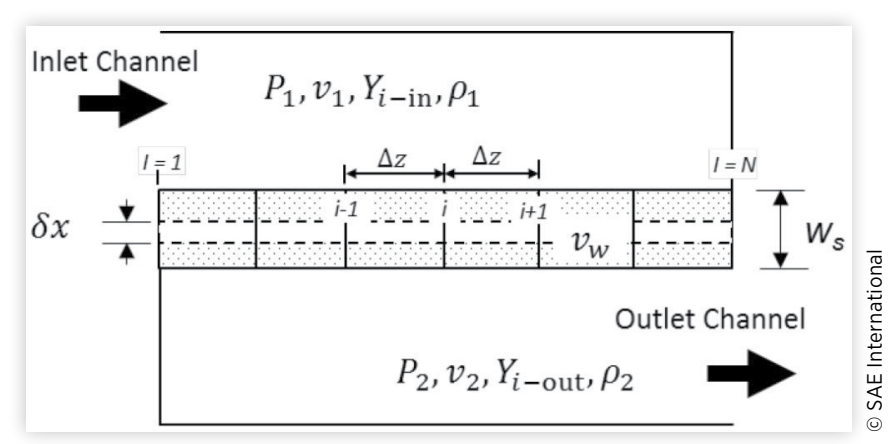

The continuity and momentum balance of the channel gas is given by equations 1 and $\underline{2}^{1}$.

$$
\begin{gathered}
\frac{\partial}{\partial z}\left(\rho_{i} v_{i} d_{i}^{2}\right)=(-1)^{i} 4 d \rho_{w} v_{w} \\
\frac{\partial p_{i}}{\partial z}+\frac{\partial}{\partial z}\left(\rho_{i} v_{i}^{2}\right)=\frac{-\gamma_{1} \mu v_{i}}{d_{i}^{2}}
\end{gathered}
$$

The total pressure drop is given in Eq. 3 .

$$
\begin{gathered}
p_{1}-p_{2}=\Delta p_{\text {channel }}+\Delta p_{\text {wall }} \\
\Delta p_{\text {wall }}=\frac{\mu v_{w}}{k_{s}} w_{s}
\end{gathered}
$$

The channel pressure drop is covered in Eq. 2, and the wall pressure drop is covered by Darcy's law (Eq. 4). Channel entrance and exit pressure losses and the Forchheimer term associated with the wall pressure drop are ignored.

Conservation of energy is given by Eq. 5 (inlet channel) and Eq. 6 (outlet channel) and Eq. 7 (wall layer). The source terms in wall energy balance are defined in Eqs. 8-10.

$$
\begin{gathered}
\left.C_{p, g} \rho_{1} v_{1}\right|_{z} \frac{\partial T_{1}}{\partial z}=h_{1} \frac{4}{d}\left(T_{s}-T_{1}\right) \\
\left.C_{p, g} \rho_{2} v_{2}\right|_{z} \frac{\partial T_{1}}{\partial z}=\left(h_{2}+C_{p, g} \rho_{w} v_{w}\right) \frac{4}{d}\left(T_{s}-T_{2}\right) \\
\rho_{s} C_{p, s} \frac{\partial T_{s}}{\partial t}=\lambda_{s, z} \frac{\partial^{2} T_{s}}{\partial x^{2}}+H_{\text {conv }}+H_{\text {wall }}+H_{\text {react }} \\
H_{\text {conv }}=h_{1} S_{F}\left(T_{1}-T_{s}\right)+h_{2} S_{F}\left(T_{2}-T_{s}\right) \\
H_{w a l l}=C_{p, g} \rho_{w} v_{w} S_{F}\left(T_{1}-T_{s}\right) \\
H_{\text {react }}=S_{F} \sum_{k}\left(\int_{-w_{p}}^{w_{w}} f_{w} R_{k} \mathrm{~d} w\right) \cdot \Delta H_{k}
\end{gathered}
$$

\footnotetext{
${ }^{1}$ Equation terms are defined in the Definitions / Abbreviations at the end of document.
} 
TABLE 1 SCR reactions scheme implemented in the model [17]

\begin{tabular}{|c|c|c|c|}
\hline $\mathbf{S} / \mathbf{N}$ & Chemical Reaction & Reaction Rate Expression & Description \\
\hline 1 & $\mathrm{~S}+\mathrm{NH}_{3} \rightarrow\left(\mathrm{NH}_{3}\right)_{S}$ & $R_{1}=k_{1} C_{N H 3}(1-\theta)$ & $\mathrm{NH}_{3}$ adsorption \\
\hline 2 & $\left(N_{3}\right)_{S} \rightarrow S+N H_{3}$ & $R_{2}=k_{2} \theta$ & $\mathrm{NH}_{3}$ desorption \\
\hline 3 & $2\left(\mathrm{NH}_{3}\right)_{S}+\mathrm{NO}+\mathrm{NO}_{2} \rightarrow 2 \mathrm{~N}_{2}+3 \mathrm{H}_{2} \mathrm{O}+2 \mathrm{~S}$ & $R_{3}=k_{3} \theta C_{N O} C_{N O 2}$ & Fast SCR \\
\hline 4 & $4\left(\mathrm{NH}_{3}\right)_{S}+4 \mathrm{NO}+\mathrm{O}_{2} \rightarrow 4 \mathrm{~N}_{2}+6 \mathrm{H}_{2} \mathrm{O}+4 \mathrm{~S}$ & $R_{4}=k_{4} \theta C_{N O}$ & Standard SCR \\
\hline 5 & $4\left(\mathrm{NH}_{3}\right)_{\mathrm{S}}+3 \mathrm{NO}_{2} \rightarrow 3.5 \mathrm{~N}_{2}+6 \mathrm{H}_{2} \mathrm{O}+4 \mathrm{~S}$ & $R_{5}=k_{5} \theta C_{N O 2}$ & Slow SCR \\
\hline 6 & $2\left(\mathrm{NH}_{3}\right)_{S}+1.5 \mathrm{O}_{2} \rightarrow \mathrm{N}_{2}+3 \mathrm{H}_{2} \mathrm{O}+2 \mathrm{~S}$ & $R_{6}=k_{6} \theta C_{02}$ & $\mathrm{NH}_{3}$ oxidation \\
\hline 8 & $\mathrm{NO}+0.5 \mathrm{O}_{2} \leftrightarrow \mathrm{NO}_{2}$ & $R_{8}=k_{8}\left[\left(C_{\mathrm{NO}} \sqrt{C_{\mathrm{O}_{2}}}\right)-\frac{C_{\mathrm{NO}_{2}}}{K_{c}}\right]$ & $\mathrm{NO}-\mathrm{NO}_{2}$ redox equilibrium \\
\hline
\end{tabular}

The specie conservation in the wall layer is given by Equation 11.

$$
v_{w} \frac{\partial y_{j}}{\partial x}-D_{j} \frac{\partial}{\partial x}\left(f_{x} \frac{\partial y_{j}}{\partial x}\right)=\frac{f_{x}}{c_{m}} \sum_{j} s_{k j} R_{j}
$$

The boundary conditions are Eq. 12 at the inlet channelwall interface, and Eq. 13 at the outlet channel-wall interface.

$$
\begin{gathered}
v_{w} y_{1 s, j}-\left.D_{j} f_{-w} \frac{\partial y_{j}}{\partial x}\right|_{1 s}=v_{w} y_{1, j}-k_{1 m, j} f_{-w}\left(y_{1 s, j}-y_{1, j}\right) \\
-\left.D_{j} \frac{\partial y_{j}}{\partial x}\right|_{2 s}=k_{2 m, j}\left(y_{2 s, j}-y_{2, j}\right)
\end{gathered}
$$

where

$$
\begin{gathered}
f_{x}=\frac{b(x)}{d} \\
b(x)= \begin{cases}d+2 w & \text { if } x<0 \\
d & \text { if } x \geq 0\end{cases}
\end{gathered}
$$

The specie conservation in the inlet and outlet channels are given by Eqs. 16 and $\underline{17}$, respectively.

$$
\begin{aligned}
& \frac{\partial}{\partial z}\left(v_{1} y_{1, j}\right)=-\frac{4}{d f_{w}^{2}} v_{w} y_{1, j}+\frac{4}{d f_{w}} k_{1 m . j}\left(y_{1 s, j}-y_{1, j}\right) \\
& \frac{\partial}{\partial z}\left(v_{2} y_{2, j}\right)=\frac{4}{d f_{w}^{2}} v_{w} y_{2, j}+\frac{4}{d f_{w}} k_{2 m . j}\left(y_{2 s, j}-y_{2, j}\right)
\end{aligned}
$$

The boundary conditions associated with the channel species balance equations are Eqs. 18 and $\underline{19}$

$$
\begin{gathered}
y_{1}(z=0)=y_{1-\text { in }} \\
y_{2}(z=0)=y_{2 s}(z=0)
\end{gathered}
$$

The rate of reaction term in the specie wall equation (Eq. 11) is given by the Eiley-Rideal rate law [15]:

$$
R_{j}=k_{j} \prod_{j} C_{g j}^{\alpha_{j}}\left(\theta \Omega_{k}\right)
$$

where the reaction rate constant $k_{j}$ is of the Arrhenius form $A_{j} e^{-E_{a, j} / R T}$. The kinetic parameters $\left(A_{j}\right.$ and $\left.E_{a, j}\right)$ are obtained from ref [16] for this work. These parameters will be calibrated following future validation of our model. The rate of reaction is defined in the units mole of component reacted per volume of reactor per time.

The SCR reaction scheme implemented in this system is presented in Table 1.

Two active sites (S1 \& S2) with different activation energy levels are considered available on the catalyst surface for $\mathrm{NH}_{3}$ storage. The dynamics of ammonia coverage on each catalyst site is given by Equation 21 .

$$
\Omega_{k} \frac{d \theta_{k}}{d t}=\sum_{j=1}^{n r} s_{k j} r_{j}\left(T_{s}, C_{s}, \theta_{k}\right)
$$

\section{Model Solution}

The model solution approach is presented in Figure 2. The solution domain is discretised in the axial and transverse direction. A method of lines approach is applied in each dimension to convert the partial differential equations to ordinary differential equations. The gas velocity field is solved in the approach of Premchand et al. [11]. Finite difference is applied for the solution of the channel species concentration and energy balances. A time-efficient algorithm which rigorously evaluates the reaction and diffusion contributions throughout the wall sub-layer is applied to solve the wall transport equation. A fourth order Runge-Kutta method is applied to solve the first-order surface coverage and wall temperature equations in time. MATLAB is used as the solution environment.

\section{Proper Orthogonal Decomposition}

Proper orthogonal decomposition (POD), also known as principal component analysis (PCA), or Karhunen-Loéve decomposition (KLD), is a statistical method which achieves model order reduction (MOR) by extracting the dominant modes of the system and using those modes to devise a lower dimensional approximation. The main idea of the POD method is 
FIGURE 2 Flow diagram of the SCR-in-DPF model solution algorithm

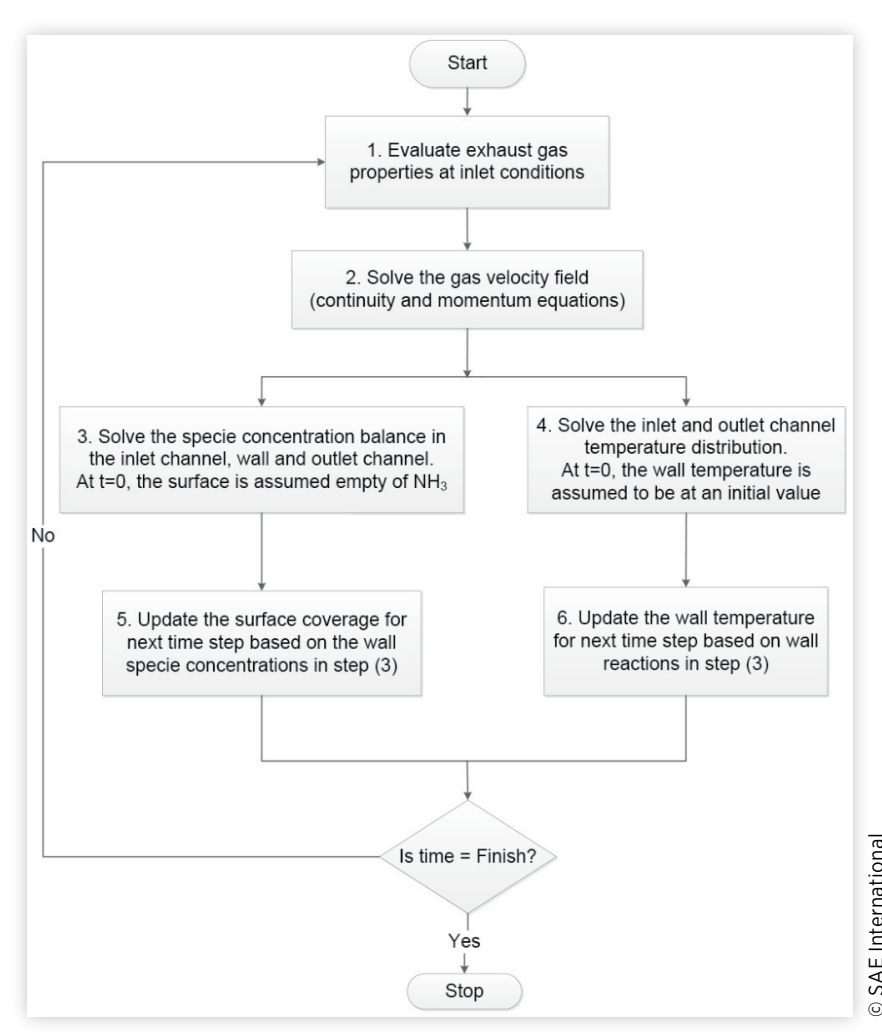

to decompose system data obtained in the course of experiment or numerical simulation into linear combination of basis functions (POD modes) and associated coefficients. Model order reduction is then achieved by Galerkin projection of the full order model on the subspace spanned by the lower-dimensioned POD modes 2 .

In this section a brief outline of the mathematical formulation of the POD method is presented with comments on the computation of the POD modes and the POD-Galerkin projection. Thereafter the POD method is applied to the 1D + 1D SCR-in-DPF model.

\section{Mathematical Formulation}

The presentation here is limited to provide sufficient background for the discussion that follows. The interested reader is referred to $[\underline{18}, \underline{19}, \underline{20}]$ for further details. The mathematical formulation below follows the presentation in reference [19].

Let $g(x, t)$ be a function over some domain of interest $\mathcal{Q}$ . At time $t_{k}$, the system displays a snapshot $g k(x)=g\left(x, t_{k}\right)$. The POD aims at obtaining the most characteristic structure $\phi(x)$ of an ensemble of snapshots of the field $g(x, t)$. This is equivalent to finding the orthonormal basis function $\phi(x)$ that

\footnotetext{
${ }^{2}$ The POD method is closely related to the theory of Hilbert spaces [26]. If the data or functions to be approximated lie in the Hilbert space, then expansion in terms of orthonormal basis functions are possible according to the following theorem: If is a separable Hilbert space with orthonormal basis, any element can be written as [22].
}

maximises the ensemble average of the inner products between $g k(x)$ and $\phi(x)$ :

$$
\max _{\phi_{i}}\left\langle\left|g^{k}, \phi\right|^{2}\right\rangle, \text { s.t. }\|\phi\|^{2}=1
$$

where $\langle f, h\rangle=\int_{\mathcal{Q}} f(x) h(x) d \mathcal{Q}$ denotes the inner product in $\mathcal{Q} ;\langle\cdot\rangle$ denotes the averaging operation; $\|\cdot\|=\left\langle\|_{\bullet} \cdot\right\rangle^{\frac{1}{2}}$ denotes the norm; and $|\cdot|$ denotes the modulus.

The constrained optimisation of Equation 22 leads to the following integral eigenvalue problem [19].

$$
\int_{\mathcal{Q}}\left\langle g^{k}(x), g^{k}\left(x^{\prime}\right)\right\rangle \phi\left(x^{\prime}\right) d x^{\prime}=\lambda \phi(x)
$$

where $\left\langle g k(x), g k\left(x^{\prime}\right)\right\rangle$ is the average auto-correlation function.

The POD modes of the function $g(x, t)$ given by $\phi(x)$ are the orthogonal eigenvectors of the integral Equation 23. The POD modes may then be used as basis for the decomposition of $g(x, t)$ :

$$
g(x, t)=\sum_{i=1}^{k} a_{i}(t) \phi_{i}(x)
$$

where the coefficients are uncorrelated, i.e. $\left\langle a_{i}(t), a_{j}(t)\right\rangle=$ $\delta_{i j} \lambda_{i}$, and are determined by $a_{i}(t)=\left\langle g(x, t), \phi_{i}(x)\right\rangle$.

The eigenvalues $\left(\lambda_{j}\right)$ of Equation 23 determine the magnitude of the POD modes. The POD mode associated with the largest eigenvalue is the optimal vector to characterise the ensemble of snapshots. The POD mode associated with the second largest eigenvalue is the optimal vector to characterise the ensemble of snapshot restricted to the space orthogonal to the first POD mode, and so forth [20].

The POD approach aims to use few POD modes associated with the $M$-largest eigenvalues to approximate the field $g(x, t)$ as per Eq. 25 .

$$
\hat{g}(x, t) \approx \sum_{i=1}^{M} a_{i}(t) \phi_{i}(x) \text { where } M \ll k
$$

\section{Computation of the POD}

Consider a system of $n$ observations of a $m$ dimensional vector, $X \in \mathbb{R}^{m \times n}$. The POD modes can be computed by performing the singular value decomposition (SVD) of the matrix $X$.

$$
X=U \Sigma V^{T}
$$

where $\mathrm{U}$ is the $m \times m$ orthogonal left-matrix, $\mathrm{V}$ is an orthogonal $n \times n$ right-matrix, and $\Sigma$ is a diagonal matrix of the singular values of $X, \sigma_{i}$. The elements of $\Sigma_{i i}$ are arranged in decreasing order, i.e. $\sigma_{1} \geq \sigma_{2} \geq \sigma_{3} \cdots \geq \sigma_{r}>0$.

If $r=\min (m, n)$ is the order of the full system, for any $p<r$, the matrix $\Sigma_{p}$ obtained by setting $\sigma_{p+1}=\sigma_{p+2}=\sigma_{p+3} \cdots$ $=\sigma_{r}=0$ in $\Sigma$ can be used to calculate a low-order and optimal rank approximation of $X$. 


$$
X_{p}=U \Sigma_{p} V^{T}
$$

The optimality of the approximation is due to the fact that the rank matrix $\Sigma_{p}$ minimises the distance between $X$ and $X_{p}$, a discrete form corollary of the maximisation definition of Equation 22. It is low-order because $p \ll r$.

The full order system POD modes are the columns of $U$, while the low-order system POD modes are the first $p$ columns of $U$. The choice of $p$ is given by the extent of variation energy that one intends to capture from the full order system as follows:

If the total system energy is the sum of the singular values $\sum_{j}^{r} \sigma_{j}$ of matrix $\Sigma$, then the energy associated with the first p-POD modes is defined in Equation 28.

$$
\varepsilon=\frac{\sum_{j=1}^{p} \sigma_{j}}{\sum_{j=1}^{r} \sigma_{j}}
$$

The low-order system POD modes can be written as an $m \times p$ orthonormal matrix $\Phi=\left[\phi_{1}, \phi_{2}, \ldots, \phi_{p}\right]$.

\section{POD-Galerkin Projection}

The Galerkin projection is a one of the mean weighted residuals method for the solution of differential equations [21]. In the weighted residuals method, the mean of the residual (defined as the difference in the solution and its approximation) weighted by an appropriate 'trial function' is forced to zero. The traditional Galerkin method uses simple continuous piecewise function as the trial functions. In the POD-Galerkin method, the POD modes are used as the trial functions. The low-order \& optimality properties of the first $p$-POD modes carry into the POD-Galerkin projection to achieve a low-order and optimal model description of the system.

Consider a full order system for the evolution of $\boldsymbol{X} \in R^{m \times n}$ in time:

$$
\frac{d}{d t} X(t)=\mathcal{L}(X(t))+\mathcal{N}(X(t))
$$

If it is possible to obtain $n$-snapshots of the evolution of $\boldsymbol{X}$ and extract the first $p$ POD modes in a POD matrix, $\Phi \in R^{m \times p}$ as described in previous section, the reduced order model can be obtained by POD-Galerkin projection as follows.

Let $\hat{X}$ be an approximation of $\boldsymbol{X}$, described by equation

$$
\frac{d}{d t} \hat{X}(t)=\mathcal{L}(\hat{X}(t))+\mathcal{N}(\hat{X}(t))+R(X)
$$

Let $R(\boldsymbol{X})$ be the residual (difference between Equations 29 and $\underline{30}$ ). The basic idea of the Galerkin projection is that given an orthonormal basis $\left\{\phi_{i}\right\}_{i=1}^{\infty}$, of a Hilbert space, the projection of the residual to the span of the first $p$ basis functions varnishes. i.e. $\left\langle\phi_{i}, R\right\rangle=0 ; i=1, \ldots, p$ or $\Phi^{T} R=0$ (for discrete systems) [르].
With the POD modes as basis functions and an approximation of $\boldsymbol{X}$ defined as $\hat{X}=\Phi \mathbf{a}$ where $\mathbf{a} \in \mathbb{R}^{n \times p}$ is the matrix of coefficients as per Equation 24, the POD-Galerkin projection is equivalent to

$$
\Phi^{T} \frac{d}{d t} \widehat{X}(t)=\Phi^{T} \mathcal{L}(\widehat{X}(t))+\Phi^{T} \mathcal{N}(\widehat{X}(t))
$$

or

$$
\Phi^{T} \Phi \frac{d}{d t} \mathbf{a}(t)=\Phi^{T} \mathcal{L}(\Phi \mathbf{a}(t))+\Phi^{T} \mathcal{N}(\Phi \mathbf{a}(t))
$$

Since $\Phi$ is orthonormal $\Phi^{T} \Phi=\mathrm{I} \in \mathbb{R}^{p \times p}$, the identity matrix. Therefore Equation 32 reduces to

$$
\frac{d}{d t} \mathbf{a}(t)=\Phi^{T} \mathcal{L}(\Phi \mathbf{a}(t))+\Phi^{T} \mathcal{N}(\Phi \mathbf{a}(t))
$$

for the evolution of a coefficients, with initial condition $\mathbf{a}(0)=\Phi^{T} \boldsymbol{X}(0)$.

Equation 33 is the Galerkin-projected reduced order form of Equation 29 based on the first $p$ POD modes $\Phi$. The approximated values of $\boldsymbol{X}$ is obtained from the POD matrix $\Phi$ and the a coefficients as per $\widehat{X}=\Phi \mathbf{a}$.

The error between the actual and approximated values of $X$ by the first $p$ POD modes is of the order of $\sum_{i=p+1}^{r} \sigma_{i}^{2}$ which is minimum by definition of the POD method.

\section{POD Application to SCR-in-DPF Model}

The first step in the application of the POD method is to obtain an ensemble data describing the system. The data can be obtained from experiments or from numerical simulation of the full order model. In this work, our system data was obtained from a numerical simulation of the SCR-in-DPF model described in the previous section. For each of the cases considered, the numerical model is solved at the specified inlet conditions, to generate the system ensemble data for the POD basis. To aid our discussion, the SCR-in-DPF model described is considered to be our high fidelity model or HFM, and the low-order POD-derived model is named the POD-model.

The wall temperature and the ammonia surface coverage (ASC) are selected as state variables on which to apply the POD method because they are the explicit time-dependent variables in our system. The remaining (quasi-steady state) equations are solved at each time step with the values of the state variables obtained from the POD model evolution. In the future the mass of soot in the system will be included as a state variable when soot dynamics is included in our HFM.

Let $\theta, T_{s} \in \mathbb{R}^{N \times T}$ be the solution obtained from the solution of the SCR-in-DPF model for ASC and wall temperature respectively at $N$ discrete units of the wall layer along the length of the catalyst, over the time period $[0, T]$. The POD modes associated with the ASC and wall temperature data ensemble can be extracted as discussed in previous section. Let $\Phi_{\theta}, \Phi_{T_{s}} \in \mathbb{R}^{N \times p}$ be the matrix of the first $p$ POD modes, 
where $p$ is the number of modes need to capture a given level of energy from the data ensemble (Equation 28). The POD-Galerkin projection of the subspace spanned by the POD modes on the HFM is then as follows:

Let $\hat{\theta} \in \mathbb{R}^{N \times p}$ be the approximate $\theta$ obtained from the low-order POD matrix as:

$$
\hat{\theta}=\Phi_{\theta} \mathbf{a}_{\theta}
$$

and, $\widehat{T}_{s}$ be the approximate $T_{s}$ obtained from the loworder POD matrix as

$$
\widehat{T_{s}}=\Phi_{T_{s}} \mathbf{a}_{T_{s}}
$$

where $\mathbf{a}_{\theta}, \mathbf{a}_{T_{s}} \in \mathbb{R}^{p \times T}$ are the a-coefficient matrices.

For compactness, let $\boldsymbol{X}$ represent $\theta$ and $T_{s}$ as follows:

$$
X=\left[\begin{array}{l}
X_{1} \\
X_{2}
\end{array}\right]=\left[\begin{array}{l}
\theta \\
T_{s}
\end{array}\right]
$$

and

$$
\widehat{X}=\left[\begin{array}{l}
\widehat{X}_{1} \\
\widehat{X_{2}}
\end{array}\right]=\left[\begin{array}{c}
\hat{\theta} \\
\widehat{T_{s}}
\end{array}\right]=\left[\begin{array}{c}
\Phi_{\theta} \mathbf{a}_{\theta} \\
\Phi_{T_{s}} \mathbf{a}_{T_{s}}
\end{array}\right]=\Phi_{X} \mathbf{a}_{X}
$$

Then Equations 21 and $\underline{7}$ can be written compactly as

$$
\frac{\partial}{\partial t}[X]=\left[\begin{array}{l}
f_{1}(X, \mu) \\
f_{2}(X, \mu)
\end{array}\right]=[f(X, \mu)]
$$

where $\boldsymbol{\mu}=\left[\boldsymbol{y}_{\boldsymbol{i}}, \boldsymbol{T}_{\boldsymbol{i}}, \ldots\right]$ is the parametric vector containing other system variables.

Using the POD modes as basis functions and an approximation of $\boldsymbol{X}$ defined in Equation 37, the POD-Galerkin projection is equivalent to

$$
\frac{d}{d t} \mathbf{a}(t)=\Phi_{X}^{T}\left[f\left(\Phi_{X} \mathbf{a}_{X}, \mu\right)\right]
$$

with the initial condition of $\mathbf{a}_{X}(0)=\Phi_{X}^{T} X(0)$.

Equation 39 for $\mathbf{a}_{X} \in \mathbb{R}^{2 p \times T}$ is lower order compared to Equation 38 for $\boldsymbol{X} \in \mathbb{R}^{2 N \times 2 p}$ because $p \ll N$. The low-order approximated variable can be reconstructed from $\mathbf{a}_{X}$ over time by Equation 37. The other system variables are then obtained based on approximate $\widehat{X}$ over time.

\section{Application}

\begin{tabular}{|c|c|}
\hline Parameter & Value \\
\hline Length $[\mathrm{m}]$ & 0.152 \\
\hline Diameter [mm] & 19.18 \\
\hline Cell Density $[\mathrm{cpsi}]$ & 300 \\
\hline Wall thickness [mil] & 12 \\
\hline Channel width $[\mathrm{mm}]$ & 1.162 \\
\hline Active site S1 storage capacity $\left[\mathrm{mole} / \mathrm{m}^{3}\right]$ & 50 \\
\hline Active site $\mathrm{S} 2$ storage capacity $\left[\mathrm{mole} / \mathrm{m}^{3}\right]$ & 30 \\
\hline Material Porosity [\%] & 58 \\
\hline Mean pore size $[\mu \mathrm{m}]$ & 22 \\
\hline Catalytic coating $[-]$ & Cu-Zeolite \\
\hline
\end{tabular}

\section{System Description}

A silicon carbide ( $\mathrm{SiC}$ ) filter with copper zeolite SCR catalyst extruded within the filter walls is used for this analysis. The

\begin{tabular}{|c|c|}
\hline Parameter & Value \\
\hline Exhaust gas flow rate $[\mathrm{g} / \mathrm{s}]$ & 0.3 \\
\hline Outlet pressure $[\mathrm{Pa}]$ & 101,325 \\
\hline Inlet gas temperature $\left[{ }^{\circ} \mathrm{C}\right]$ & 200 \\
\hline Space velocity $[1 / \mathrm{h}]$ & 34,000 \\
\hline $\begin{array}{l}\text { Initial } \mathrm{NH}_{3} \text { coverage on catalyst } \\
\text { surface }\end{array}$ & 0 (i.e. empty) \\
\hline Feed gas composition & 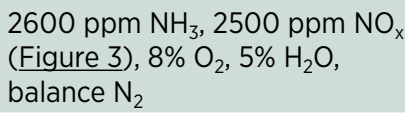 \\
\hline
\end{tabular}
specification of the SCR-filter is presented in Table 2.
TABLE 2 SCR Filter Specification

TABLE 3 Inlet gas condition for the steady state NOx conversion simulation

FIGURE 3 Feed gas $\mathrm{NH}_{3}$ and $\mathrm{NO}_{x}$ concentrations.

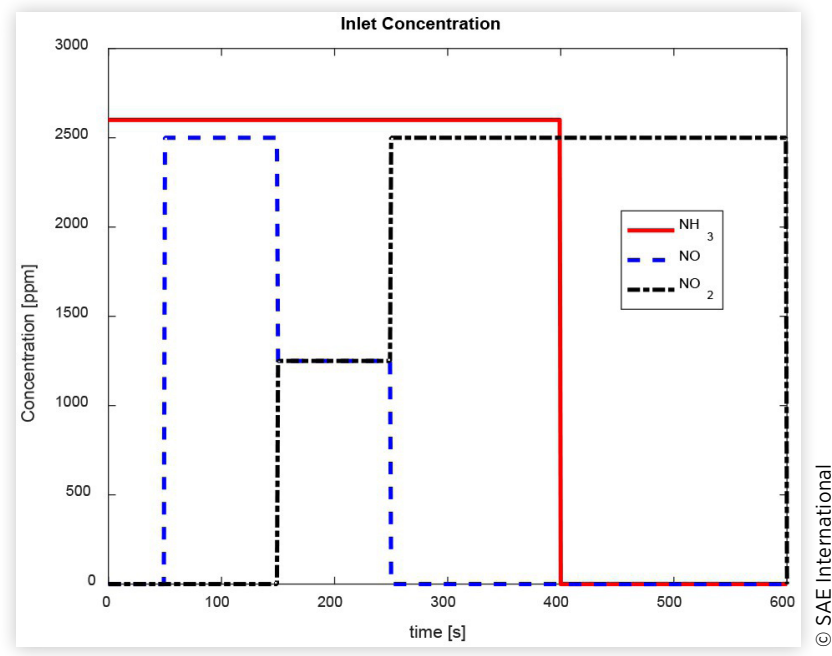

\section{Steady State $\mathrm{NO}_{x}$ Conversion}

The performance of the POD method on the system at steady state is presented in this section. The inlet condition for this scenario is presented in Table 3 . The system temperature is taken to be fixed at the inlet gas condition. The feed gas $\mathrm{NH}_{3}$ and $\mathrm{NO}_{\mathrm{x}}$ concentration over time is presented in Figure 3. 
FIGURE 4 Singular values (a) and relative significance of POD-modes (b) of the ASC $(\theta)$ data, steady state $\mathrm{NO}_{\mathrm{x}}$ conversion case

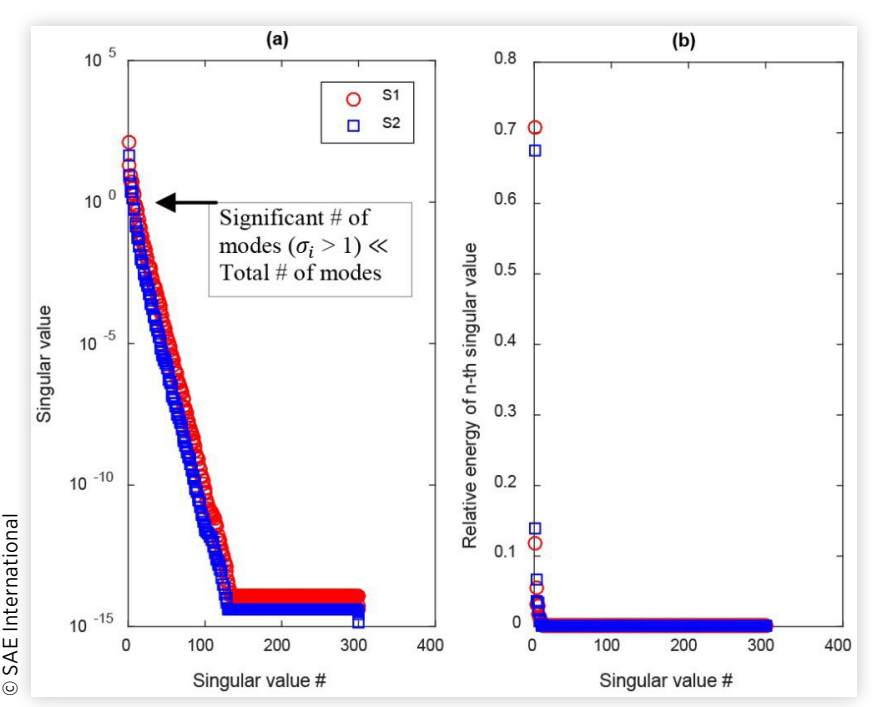

FIGURE 5 Plot of first few POD basis functions of the $\theta$ data, steady state $\mathrm{NO}_{\mathrm{x}}$ conversion case

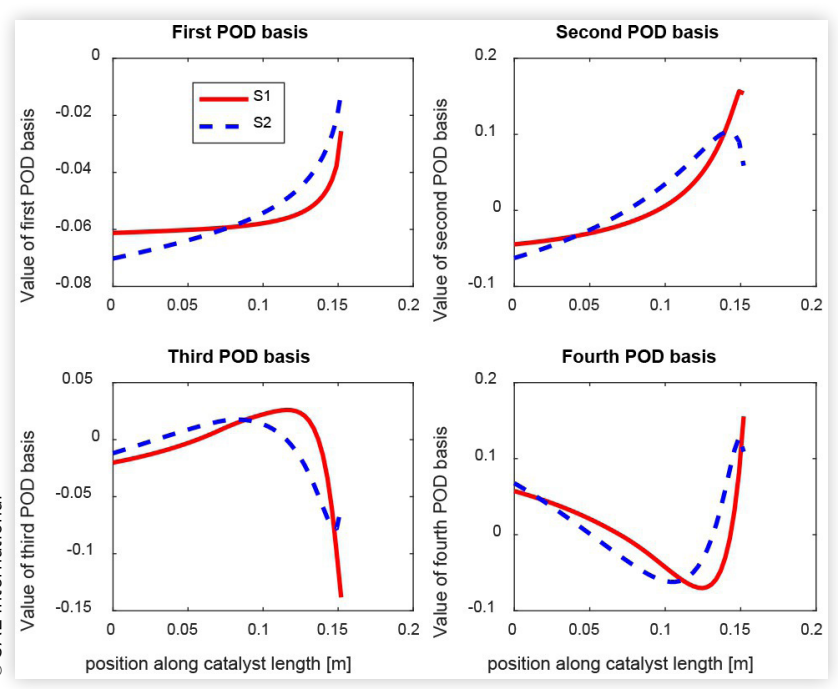

POD Modes The spectrum of the singular values obtained from the decomposition of the ASC data ensemble is presented in Figure 4. It is obvious from the plot that only a few POD modes corresponding to the largest singular values are dominant (compare the number of modes with singular values greater than 1 to the rest). The relative energy plot also shows that the first few modes combine to capture over $99 \%$ of energy of the data. These therefore indicate that the ASC dataset can be represented by the few largest POD modes without loss of data fidelity.

The first few POD modes of the ammonia surface coverage ensemble data is presented in Figure 5.

The POD modes do not necessarily have physical meanings [20].
FIGURE 6 Approximate outlet $\mathrm{NO}_{x}$ and $\mathrm{NH}_{3}$ concentration from POD-Galerkin analysis for the first four POD modes, steady state $\mathrm{NO}_{x}$ conversion case

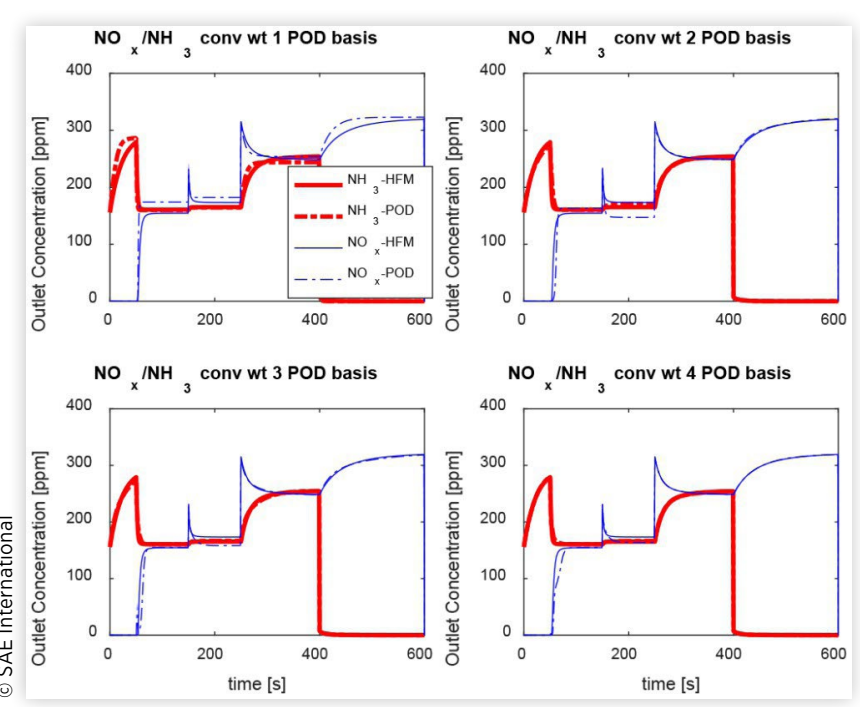

Comparative Results The $\mathrm{NO}_{x}$ and $\mathrm{NH}_{3}$ conversion predicted by the HFM and POD models are shown in Figure $\underline{6}$ for the first four POD modes. The relative errors in the prediction and summary of additional results are presented in Table 4.

It can be seen that the prediction of our POD model agrees very closely with the HFM. This attests in general to the application of POD-Galerkin technique for the solution of the model SCR-in-DPF equation. Furthermore it is obvious that the POD model based on the first four POD modes satisfactorily captures the system dynamics in this scenario as the relative error in prediction is in the order of $1-3 \%$ in comparison with the HFM.

In general the relative error reduces with the incorporation of additional POD modes. By definition of the POD approach, more POD modes will achieve a better approximation of the original dataset (Eq. 25). The POD method is attractive because it can achieve a good approximation with only a few POD modes, and thereby achieve model order reduction.

The evidence of the model order reduction achieved by the POD Galerkin technique is in the reduction in computational time to process the POD model in comparison with the HFM. (The specification of the operating PC is Intel i5 4460 processor, 3.2 GHz CPU, 32GB RAM, running MATLAB

TABLE 4 Summary of POD model performance for steady state $\mathrm{NO}_{x}$ conversion scenario

\begin{tabular}{|c|c|c|c|c|}
\hline & \multirow[b]{2}{*}{ CPU time [s] } & \multicolumn{3}{|c|}{ Relative RMS Error [\%] } \\
\hline & & ASC & $\mathrm{NO}_{\mathrm{x}}$ & $\mathrm{NH}_{3}$ \\
\hline HFM & 34.69 & & & \\
\hline 1-POD model & 15.98 & 15 & 7 & 6 \\
\hline 2-PODs model & 16.93 & 6 & 6 & 3 \\
\hline 3-PODs model & 17.26 & 2 & 5 & 2 \\
\hline 4-PODs model & 18.06 & 2 & 3 & 1 \\
\hline
\end{tabular}


FIGURE 7 Approximate ammonia surface coverage $\hat{\boldsymbol{\theta}}$ from POD-Galerkin analysis for the first four POD modes, steady state $\mathrm{NO}_{\mathrm{x}}$ conversion case

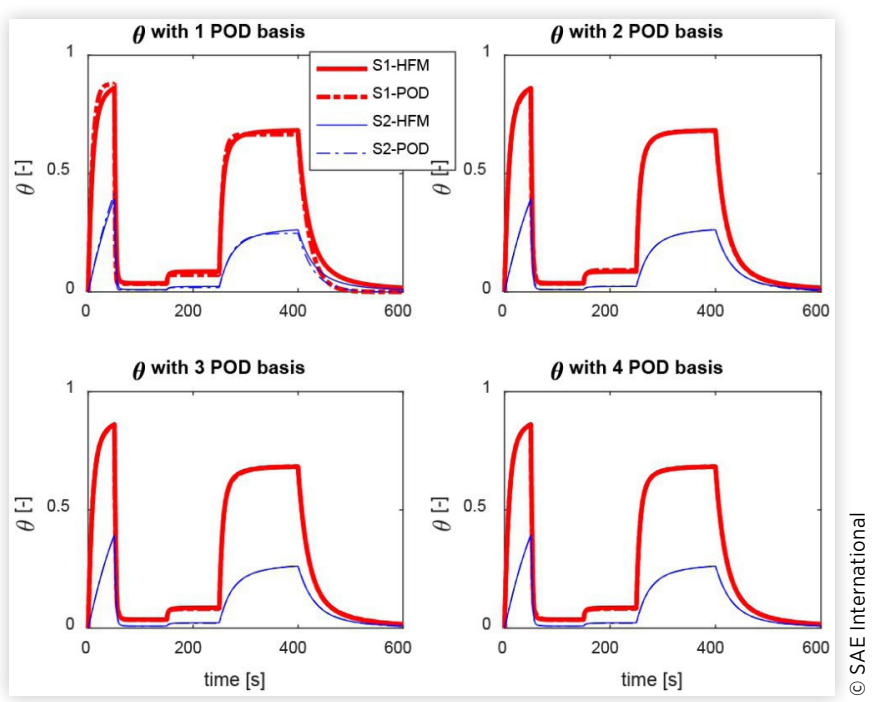

2016a; same time step of $1 \mathrm{~s}$ is specified for HFM and POD models.). In this example, nearly $50 \%$ of the computational time is saved in the POD model with 4-POD modes compared to the high fidelity model (Table 4).

The prediction of ASC for the POD model is presented in Figure 7 for the first four POD modes. It also shows that the predictive ability of the POD model closely matches the HFM with only a few POD modes.

Robustness of the POD Modes The POD modes are generated based on the simulation results for a set of inlet conditions. For the steady state case, the inlet conditions are presented in Table 3 and Figure 3 . The generated POD basis vectors are robust to deviation in the inlet conditions.

In this section, the feed gas composition in Figure 3.is excited with uniform random noise $($ mean $=1)$. The noisy inlet condition is set as input to the POD model. The POD basis vectors were generated based on the HFM results for the steady state case previously presented. The objective was to assess the performance of the POD model for inlet conditions different from that for which the POD basis vectors were generated.

The performance of the POD model (with 4 POD modes) is compared with the steady state HFM in Figure 8 and Table $\underline{5}$. In the results shown, the HFM result is for the steady state example previously presented.

It can be seen that POD model predicts the average ammonia surface coverage even with random deviation in the inlet gas conditions relatively well when compared to the HFM. The predicted output $\mathrm{NO}_{x}$ and $\mathrm{NH}_{3}$ concentrations vary randomly according to the input deviation. However, the prediction of cumulative outlet concentrations over the simulation period agrees well with the HFM with less than $1 \%$ relative error. The results show that the POD basis mode is robust to deviation in inlet conditions.

In this example the relative prediction errors of $1-3 \%$ and up to $50 \%$ savings in computation time for the POD model vs the HFM show that the POD technique with only a few modes
FIGURE 8 Outlet concentration and ammonia surface coverage for steady state POD simulation with uniform random deviation in feed gas concentration
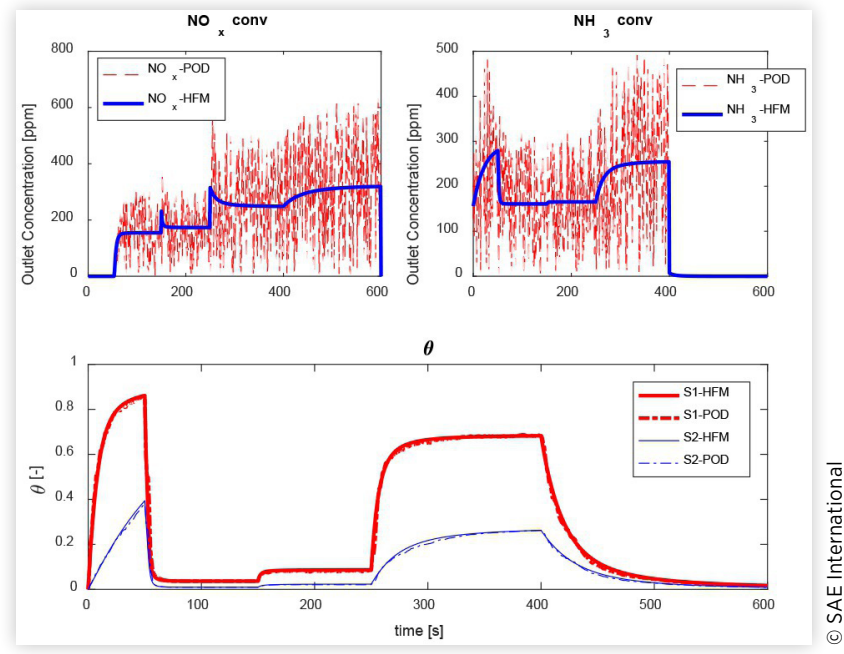

TABLE 5 Summary of POD model performance for steady state NOx conversion when uniform random deviation is applied to feed gas concentration

\begin{tabular}{|c|c|c|c|c|}
\hline & \multirow[b]{2}{*}{ CPU time [s] } & \multicolumn{3}{|c|}{ Relative RMS Error [\%] } \\
\hline & & ASC & $\mathrm{NO}_{\mathrm{x}}$ & $\mathrm{NH}_{3}$ \\
\hline HFM & 35.43 & & & \\
\hline POD model & 18.88 & 5 & $\begin{array}{l}0.6^{a} \\
63^{b}\end{array}$ & $\begin{array}{l}0.28^{a} \\
70^{b}\end{array}$ \\
\hline
\end{tabular}

a denotes error in cumulative outlet concentration

b denotes error in instantaneous outlet concentration

can deliver a low order model that preserve a high degree of fidelity. The generated POD modes are also robust to uniform random deviation in inlet conditions thus preventing the need to regenerate the POD basis vector with slight change in inlet conditions.

\section{Transient State $\mathrm{NO}_{x}$ Conversion}

The performance of the POD method on the system during a transient state operation is presented in this section.

Transient input conditions are obtained from a sample world harmonized test cycle (WHTC) conditions reported in ref [23]. The engine-out $\mathrm{NO}_{\mathrm{x}}$ emission concentrations and the exhaust gas temperatures are obtained from the reference ${ }^{3}$. Details of the test engine conditions can be found in ref [23]. The exhaust gas flow rate was constant for the simulation. In the experiment, liquid urea solution was injected, thus the input $\mathrm{NH}_{3}$ gas concentration was not measured. For our analysis, in the first 30 seconds gaseous $\mathrm{NH}_{3}$ at 1500 ppm is injected, thereafter $\mathrm{NH}_{3}$ rate at $\mathrm{ANR}=1.1$ is used. The inlet $\mathrm{NO}_{\mathrm{x}}$ and $\mathrm{NH}_{3}$ concentrations and exhaust gas temperatures are presented in Figure 9. Other input conditions are as per Table 3.

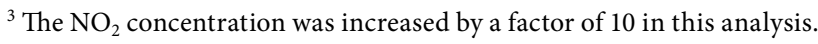

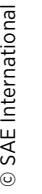

.


FIGURE 9 Engine-out specie concentrations and exhaust gas temperature from ref [23] used as input in transient analysis
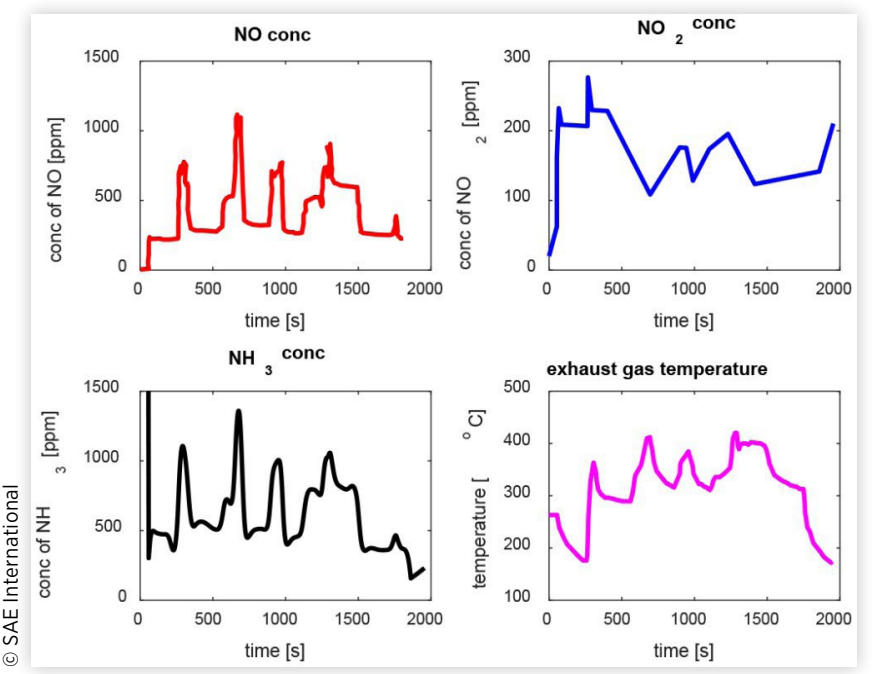

FIGURE 10 Singular values (a) and relative significance of POD-modes (b) of the ASC $(\theta)$ data, Transient state $\mathrm{NO}_{x}$ conversion case.

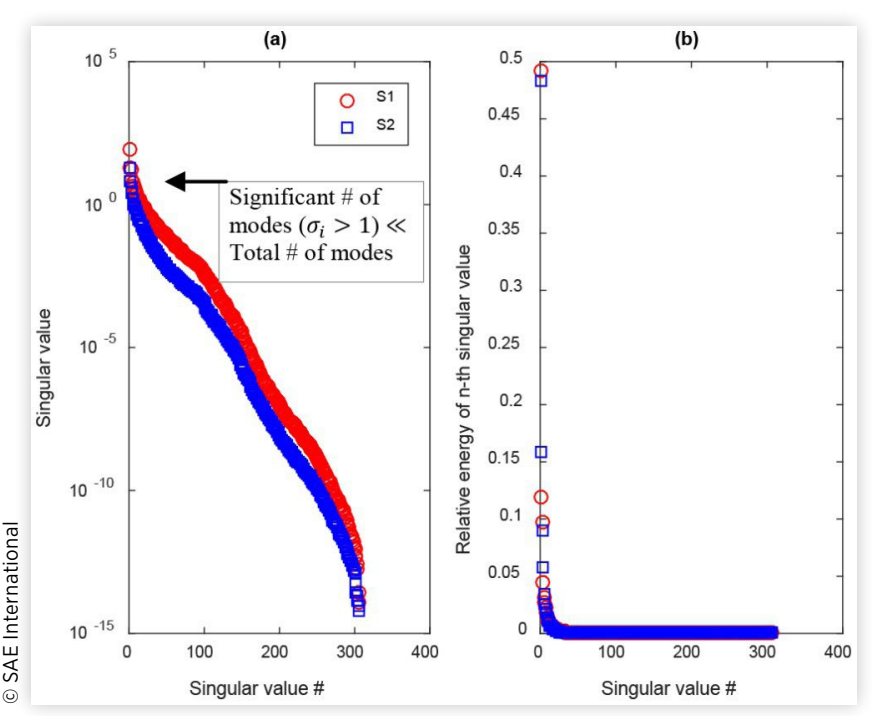

POD Modes The ammonia surface coverage and wall temperature are used as the system states in the transient $\mathrm{NO}_{\mathrm{x}}$ conversion analysis. The POD modes for the ammonia surface coverage and wall temperature dataset are presented in Figures $\underline{10}$ and $\underline{11}$, respectively.

The main observation is similar to the steady state case. A relatively few POD modes dominate the dataset, thus the POD-Galerkin technique can be applied to achieve model order reduction using the first few POD modes of the datasets. The first four POD basis functions are presented for the ASC and wall temperature dataset in Figure 12. No physical meaning can necessarily be derived from the form of the POD basis functions [20].
FIGURE 11 Singular values (a) and relative significance of POD-modes (b) of the wall temperature $\left(T_{s}\right)$ data, transient state $\mathrm{NO}_{x}$ conversion case.

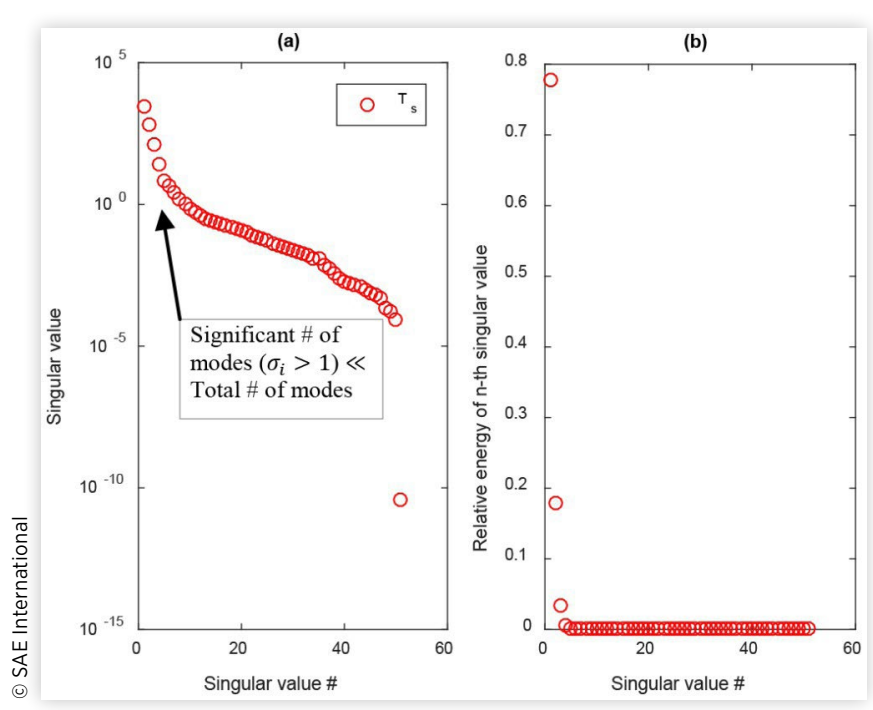

FIGURE 12 Plot of first few POD basis functions of the $\theta$ and $\mathrm{T}_{\mathrm{s}}$ data, transient state $\mathrm{NO}_{\mathrm{x}}$ conversion case

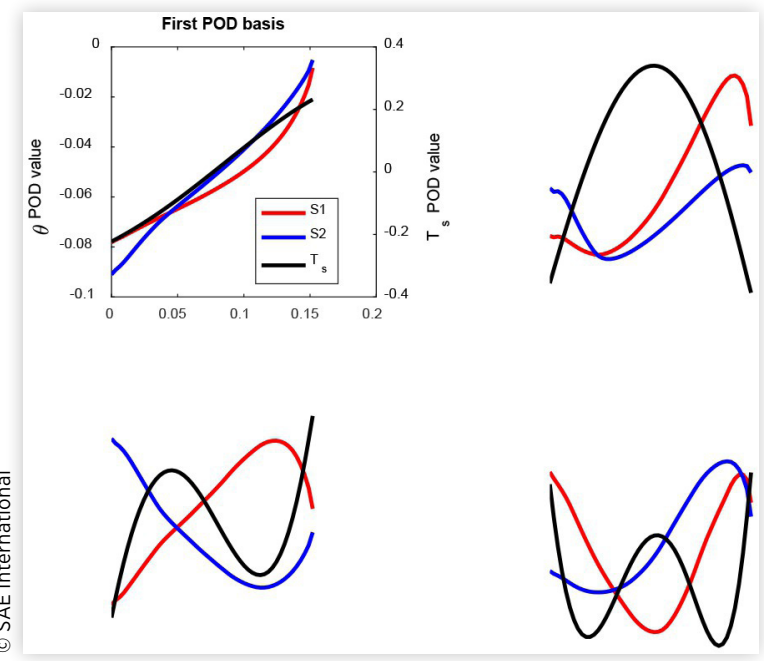

Comparative Results The $\mathrm{NO}_{\mathrm{x}}$ and $\mathrm{NH}_{3}$ conversion for the HFM and POD models are compared in Figure 13. The relative errors and CPU simulation times are presented in Table 6.

The performance of the POD model agrees well with the HFM for $\mathrm{NO}_{x}$ and $\mathrm{NH}_{3}$ conversion. In this scenario, about 15 POD modes are required in the POD model to closely match the $\mathrm{HFM} \mathrm{NO}$ conversion prediction. The relative error in $\mathrm{NO}_{\mathrm{x}}$ conversion prediction between the HFM and the POD model with 15 POD modes is ca 7\% (Table 6). More POD modes are required to more closely match the HFM prediction of outlet NOx concentration in this scenario because of the transient nature of the problem. However, 15 POD modes still 
FIGURE 13 Approximate outlet $\mathrm{NO}_{x}$ and $\mathrm{NH}_{3}$ concentration from POD-Galerkin analysis for the first few POD modes, transient state $\mathrm{NO}_{x}$ conversion case

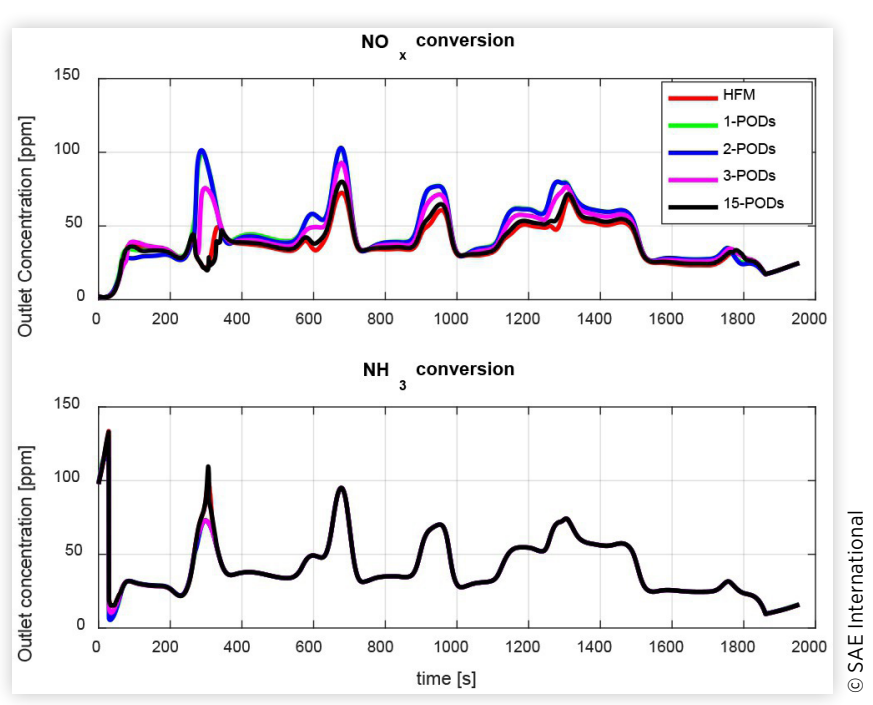

represent a significantly small proportion of the available POD modes (see Figures 10 and 11 ). Therefore model order reduction is achieved even with 15 POD modes as evident in the $40 \%$ reduction in CPU time to complete the POD model simulation compared with the HFM.

The ASC and wall temperature prediction for this scenario is presented in Figure 14.

Our system is idealized in that gaseous ammonia is used and ammonia rate is directly inferred from the inlet NOx concentration. Therefore for the majority of the simulation no excess ammonia is retained on the surface of the catalyst. In reality to preempt disturbance in the inlet NOx concentration it is prudent to have a buffer of ammonia coverage on the catalyst surface to prevent outlet NOx excursion. This will factor in the control objective in the next section on system control.

The dynamics of the wall temperature prediction is very well captured with 1 POD mode (relative error $<1 \%$, Table 6). As our system was initially warm and no soot is modelled in this analysis, the dynamics of the wall temperature is expected to follow the inlet exhaust gas temperature input as with relatively minimal energy contribution from the SCR reactions. In our follow up study, it will be interesting to see how the dynamics of wall temperature changes when soot deposition and oxidation is included in our HFM and mass of soot is added as one of our system states for the POD model.

Robustness of the POD Modes The POD modes are generated based on the simulation results for a set of inlet conditions. For the transient case, the inlet conditions are presented in Figure 9. However, the generated POD modes are robust to uniform random deviation in the inlet conditions. This section presents the performance of the POD basis vectors generated for the transient case inlet conditions applied to different inlet conditions.

A uniform random distribution (with mean of 1) is applied to the feed gas concentration to simulate different inlet
TABLE 6 Summary of POD model performance for transient state $\mathrm{NO}_{x}$ conversion scenario

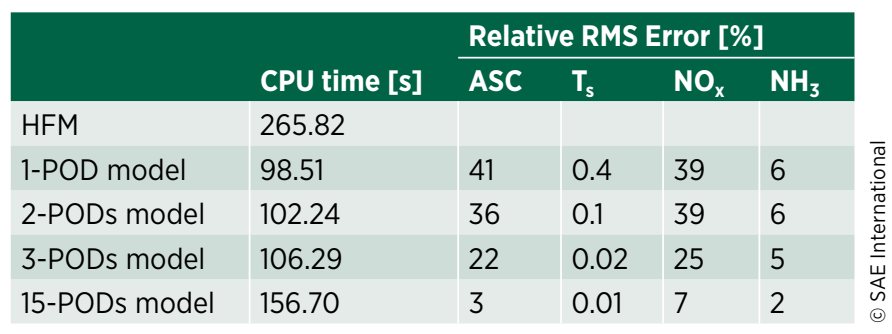

FIGURE 14 Approximate ammonia surface coverage $\hat{\boldsymbol{\theta}}$ and wall temperature $\hat{T_{s}}$ from POD-Galerkin analysis for the first few POD modes, transient state $\mathrm{NO}_{\mathrm{x}}$ conversion case
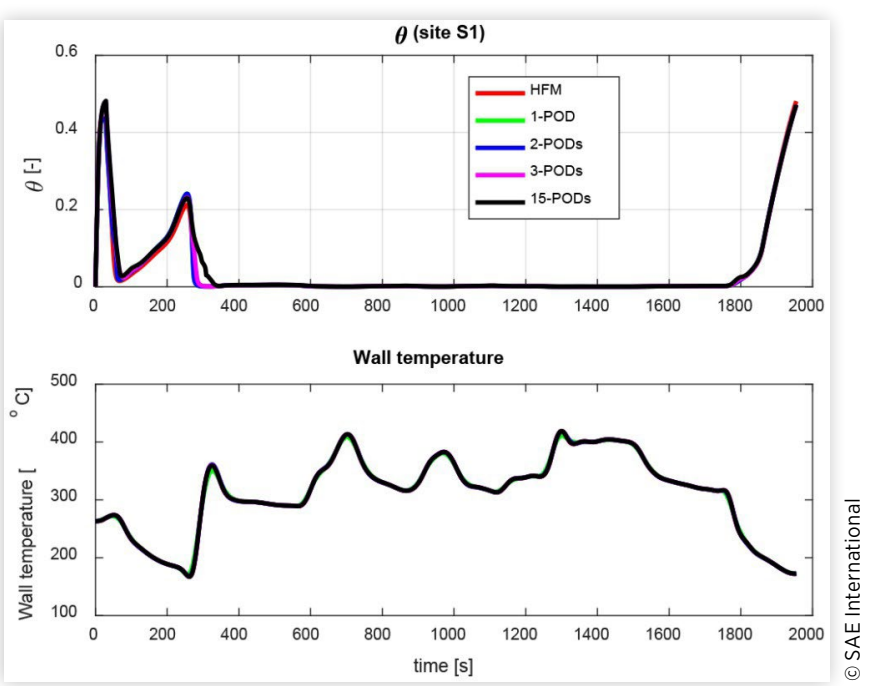

conditions. The performance of the POD model (with 15 POD modes) is compared with the HFM in Figure 8 and Table 5.

It can be seen that the POD model agrees with the HFM when the inlet feed gas concentration varies in a uniform random distribution. The outlet $\mathrm{NO}_{\mathrm{x}}$ and $\mathrm{NH}_{3}$ concentrations vary randomly according to the input deviation. The prediction of cumulative outlet concentrations agrees well with the HFM with less than 5\% relative error (Table 7). The results show that the POD basis mode is robust to uniform random deviation in inlet conditions.

For the transient analysis presented here, the POD model with 15 modes (although with a few more POD modes than in the steady state case, but still considerable less than the available total) satisfactorily matches the HFM with relative error ca $7 \%$ and less. It also achieves over $40 \%$ reduction in computational time compared to the HFM. Thus the POD model also delivers model order reduction while preserving a high degree of fidelity in this application. The POD model is also robust to uniform random deviation in inlet conditions.

\section{System Control}

POD has been applied for the model order reduction for model-based control design $[\underline{22}, \underline{24}, \underline{25}]$. We demonstrate the 
FIGURE 15 Outlet concentration and ammonia surface coverage for steady state POD simulation with uniform random deviation in feed gas concentration

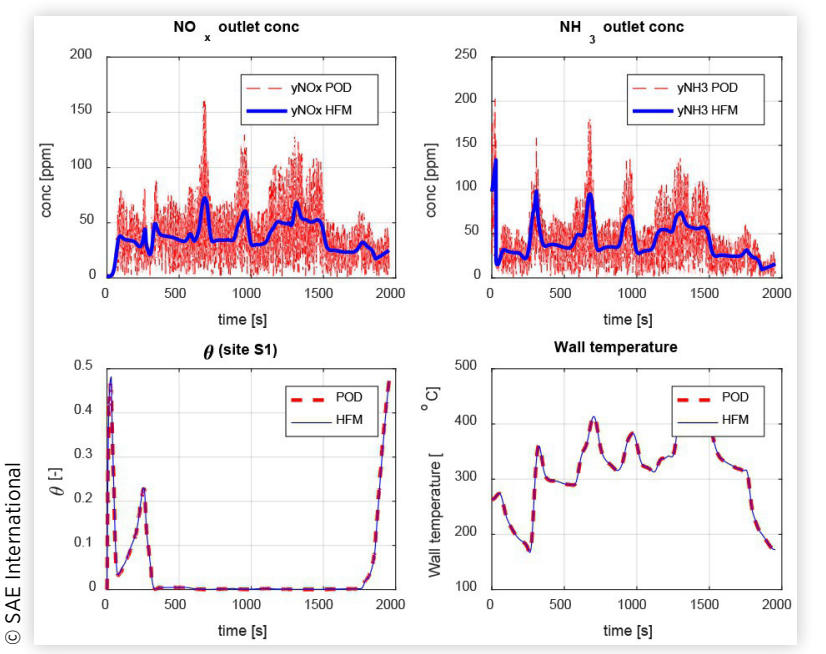

TABLE 7 Summary of POD model performance for steady state NOx conversion when uniform random deviation is applied to feed gas concentration

\begin{tabular}{|c|c|c|c|c|c|}
\hline & \multirow{2}{*}{$\begin{array}{l}\text { CPU } \\
\text { time [s] }\end{array}$} & \multicolumn{4}{|c|}{ Relative RMS Error [\%] } \\
\hline & & ASC & $T_{s}$ & $\mathrm{NO}_{\mathrm{x}}$ & $\mathrm{NH}_{3}$ \\
\hline HFM & 257.31 & & & & \\
\hline $\begin{array}{l}\text { POD } \\
\text { model }\end{array}$ & 154.58 & 5 & 0.06 & $\begin{array}{l}5 a \\
66 b\end{array}$ & $\begin{array}{l}3 a \\
63 b\end{array}$ \\
\hline
\end{tabular}

a denotes error in cumulative outlet concentration

${ }^{b}$ denotes error in instantaneous outlet concentration

application of POD in a simple control application for the SCR-in-DPF model. A simple P\&ID controller is designed for our system operating in transient condition. The controller objective is to maintain the ammonia surface coverage ${ }^{4}$ at a low level which minimizes $\mathrm{NH}_{3}$ slip but is sufficient to maximize $\mathrm{NO}_{\mathrm{x}}$ conversion.

The system state for control is taken to be the average value of ASC across the wall surface. We surmised that the 'average' is a crude approach to reduce the distribution of ASC on the surface to a scalar quantity, and applied the POD method with only the first POD mode as a comparative alternative to achieve the system state for the controller. The first POD mode is generally considered to be mean of the data ensemble [22]. Thus two controller designs were evaluated: (a) based on HFM with average ASC as system state, and (b) based on the POD model with 1 POD mode used to derive an average ASC for the system state. An open loop (OL) scenario is also simulated as a baseline for control performance evaluation. The data from the open loop run is used to generate the POD basis vectors. Controller tuning is maintained across the HFM and POD model runs to minimise system variability. The

\footnotetext{
${ }^{4}$ ASC is not measurable in practice, so an observer is typically designed to estimate ASC from the available output (outlet concentration) and system model [27]. To keep this analysis simple, ASC is obtained directly from our simulation.
}

\section{FIGURE 16 Schematic of the simple control system}

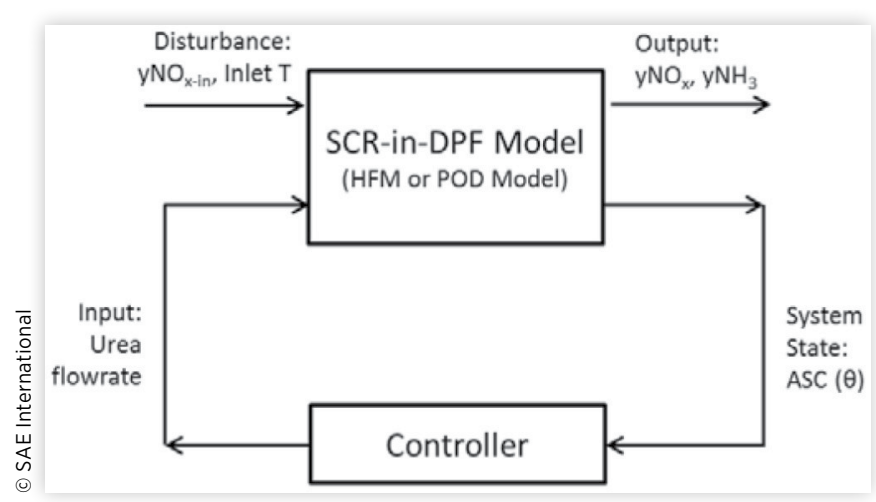

FIGURE 17 Performance of the controller based on the HFM and the PO model with only one POD mode

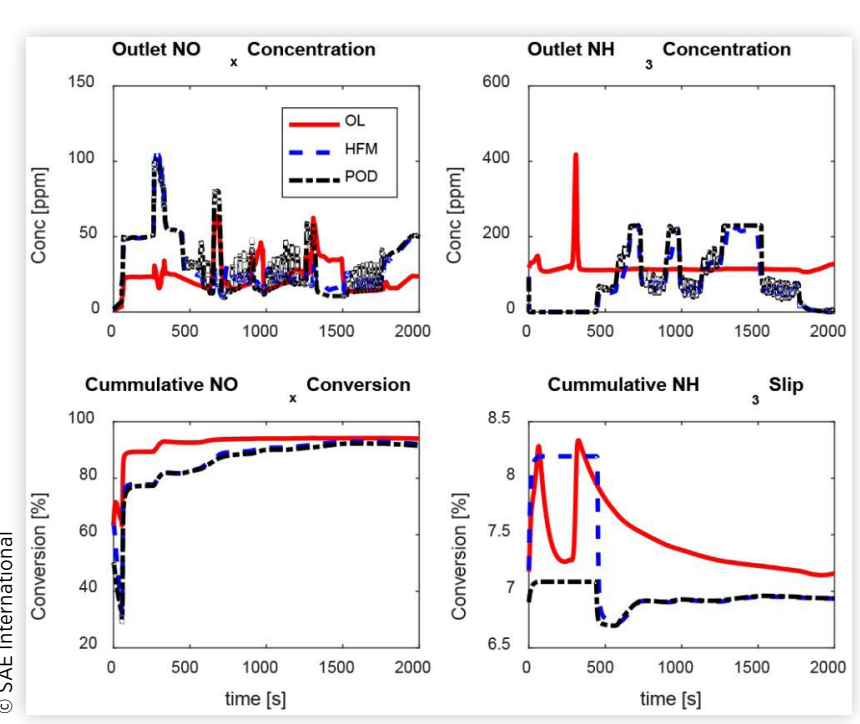

controller set point is specified at the open loop average ammonia surface coverage.

A schematic of our control system is shown in Figure 16. The inlet $\mathrm{NO}_{\mathrm{x}}$ and exhaust gas temperatures (disturbance) are the same as Figure 9. The input is configured as liquid urea flowrate. The injection valve is assumed to be able to supply up to $0.1 \mathrm{l} / \mathrm{h}$ urea solution, with instantaneous conversion of the component urea to ammonia gas. For the open loop run urea injection is fixed at half the maximum injection rate.

The controller performance based on the HFM and POD model in comparison with the open loop (OL) system response are presented in Figure 17.

The outlet $\mathrm{NO}_{\mathrm{x}}$ and $\mathrm{NH}_{3}$ concentrations trends from the HFM and POD model based controllers agree very closely. This indicates that the performance of the POD model based controller is comparable to that based on the HFM. This demonstrates the application of the POD method in this control application as an alternative to the full order model. See Table 8 for summary of other key results from the simulation.

The simulation time savings gained by applying the POD method is reflected in the performance of the control system. About 55\% reduction in simulation time is obtained when the 
TABLE 8 Summary of HFM and POD model based controller performance results

\begin{tabular}{|c|c|c|c|}
\hline & $\mathbf{O L}$ & HFM & POD \\
\hline CPU simulation time $[\mathrm{s}]$ & 284.6 & 303.1 & 137.8 \\
\hline Cumulative $\mathrm{NO}_{x}$ conversion at end [\%] & 94 & 92 & 91 \\
\hline Cumulative $\mathrm{NH}_{3}$ slip at end [\%] & 7.2 & 6.9 & 6.9 \\
\hline Total urea injected [ml] & 2.33 & 1.57 & 1.78 \\
\hline$\%$ of total urea in OL case & & 67 & 76 \\
\hline
\end{tabular}

POD-model based controller is simulated compared to the controller based on the HFM. The maximum difference in the predicted cumulative conversion at the end of simulation is ca $1 \%$. This demonstrates that the POD approach can be applied to model order reduction for control design while preserving a high degree of fidelity.

In this example it is noted that the controller only marginally changes the system performance in terms of $\mathrm{NO}_{\mathrm{x}}$ conversion and $\mathrm{NH}_{3}$ slip compared to the open loop (OL) simulation. The real difference is in the actual volume of urea used in the open loop vs. controlled scenarios. The open loop scenario consumes up to $33 \%$ more urea than the controlled scenarios. As the simulation represents an ideal system, the inlet urea rates convert directly to outlet $\mathrm{NH}_{3}$ concentration, thereby maintaining similar conversion rates with the controlled system. It is expected that this relationship is weakened under actual operating conditions, and that difference in inlet urea rate translate to variation in outlet specie conversion.

The simple control example reported here demonstrates the application of the POD method as a model order reduction technique for model-based system control design. The POD method achieves significant savings in computational time and preserves a high degree of model fidelity.

\section{Conclusions}

The POD method, with Galerkin projection, is able to deliver a low (reduced) order model which retains a high degree of fidelity. The POD method uses the dominant characteristic modes of the system as the basis for model approximation. Most dynamical systems have a few dominant modes; therefore a few POD modes can achieve satisfactory model approximation. The reduced order model relies on the same underlying physics as the full order model thereby preserves a high degree of fidelity.

The POD method was applied in this paper to a $1 D+1 D$ SCR-in-DPF model, with ammonia surface coverage and wall temperature as the system states. The results demonstrate the application of the POD-Galerkin technique to achieve model order reduction for the SCR-in-DPF model. Significant computational time savings (40-50\%) was obtained with the POD model compared with the full order model in all our simulations. Thus model order reduction was achieved. The predictions of the POD model with only a few POD modes agree closely with the full order model indicating the preservation of model fidelity. The relative errors were in the order of $1-3 \%$ for the steady state simulation, less than $7 \%$ in the transient state simulation and about $1 \%$ in the control application. Therefore the POD technique offers the promise of delivering lower order high fidelity model of the EATS which is ECU-ready.

The implementation of the POD method relies on the availability of reliable system data. This data can be obtained from simulation of the full order model for a short period to obtain an ensemble of snapshots. The POD basis vectors will depend on the inlet conditions used to generate the simulation data. In our examples, we used the ensemble data generated from the HFM runs over the entire simulation period. (It was convenient to do this as the goal was to compare the POD and HFM, and the HFM results were already available). We demonstrated that the POD basis vectors thus obtained are robust to uniform random deviation in inlet conditions. The optimal decision on how much of the simulation to run to generate robust POD basis vectors will be context specific. Factors to consider include the intent for model application, the HFM simulation speed, the nature of variations expected in the inlet conditions among others. Experimental data is a suitable alternative source for the POD method. Spatially resolved techniques will provide better quality data for obtaining the POD basis vectors.

Soot dynamics is not included in our SCR filter model, so conclusions are tentative. Our future work will apply the POD method to the SCR-in-DPF model with soot accumulation dynamics. We will compare the performance of the POD method when the POD modes are obtained from experimental data and model simulation. Furthermore system control based on the POD mode coefficients in an optimal LQR framework will be applied.

\section{References}

1. Fleming, R.D., "Fuel Economy of Light-Duty Diesel Vehicles," SAE Technical Paper 760592, 1976, doi:10.4271/760592.

2. Tang, W., Youngren, D., SantaMaria, M., and Kumar, S., "On-Engine Investigation of SCR on Filters (SCRoF) for HDD Passive Applications," SAE Int. J. Engines 6(2):862-872, 2013, doi:10.4271/2013-01-1066.

3. Tan, J., Solbrig, C., and Schmieg, S.J., "The Development of Advanced 2-Way SCR/DPF Systems to Meet Future HeavyDuty Diesel Emissions," SAE Technical Paper 2011-01-1140, 2011, doi:10.4271/2011-01-1140.

4. Olowojebutu, S. and Steffen, T., "A Review of the Literature on Modelling of Integrated SCR-In-DPF Systems," SAE Technical Paper 2017-01-0976, 2017, doi:10.4271/2017-01$\underline{0976}$.

5. Chen, H., Reuss, D.L., Hung, D.L.S., and Sick, V., "A Practical Guide for Using Proper Orthogonal Decomposition in Engine Research," Int J Engine Res 14(4):307-319, 2012, doi:10.1177/1468087412455748.

6. Fogleman, M., Lumley, J., Rempfer, D., and Haworth, D., "Application of the Proper Orthogonal Decomposition to Datasets of Internal Combustion Engine Flows," J Turbul 5(23):1-3, 2004. 
7. Alonso, D., Velazquez, A., and Vega, J.M., "A Method to Generate Computationally Efficient Reduced Order Models," Comput Methods Appl Mech Eng 198(33):2683-2691, 2009.

8. Cavendish, J.C. and Oh, S.H., "A Computationally Efficient Galerkin Technique for Approximating Transient DiffusionReaction Equations in Composite Media," Chem Eng J 17(1):41-54, 1979.

9. Oh, S.H. and Cavendish, J.C., "Transients of Monolithic Catalytic Converters. Response to Step Changes in Feedstream Temperature as Related to Controlling Automobile Emissions," Ind Eng Chem Prod Res Dev 21(1):29-37, 1982.

10. Koltsakis, G., Haralampous, O., Depcik, C., and Ragone, J.C., "Catalyzed Diesel Particulate Filter Modeling," Rev Chem Eng 29(1):1-61, 2013, doi:10.1515/revce-2012-0008.

11. Premchand, K.C., Johnson, J.H., and Yang, S.-L., "Development of a 1-D Catalyzed Diesel Particulate Filter Model for Simulation of the Oxidation of Particulate Matter and Gaseous Species during Passive Oxidation and Active Regeneration," SAE Technical Paper 2013-01-1574, 2013, doi:10.4271/2013-01-1574.

12. Tronconi, E., Nova, I., Marchitti, F., Koltsakis, G. et al., "Interaction of NO x Reduction and Soot Oxidation in a DPF with Cu-Zeolite SCR Coating," Emiss Control Sci Technol 1(2):134-151, 2015, doi:10.1007/s40825-015-0014-y.

13. Haralampous, O.A., Koltsakis, G.C., Samaras, Z.C. et al., "Reaction and Diffusion Phenomena in Catalyzed Diesel Particulate Filters," SAE Technical Paper 2004-01-0696, 2004, doi:10.4271/2004-01-0696.

14. Surenhalli, H.S., Premchand, K., Johnson, J.H., and Parker, G., "Modeling Study of Active Regeneration of a Catalyzed Particulate Filter Using One-Dimensional DOC and CPF Models," SAE Technical Paper 2011-01-1242, 2011, doi:10.4271/2011-01-1242.

15. Olsson, L. and Andersson, B., "Kinetic Modelling in Automotive Catalysis," Top Catal 28(1):89-98, 2004, doi:10.1023/B:TOCA.0000024337.50617.8e.

16. Park, S.-Y., Narayanaswamy, K., Schmieg, S.J., and Rutland, C.J., "A Model Development for Evaluating Soot-NOx Interactions in a Blended 2-Way Diesel Particulate Filter/ Selective Catalytic Reduction," Ind Eng Chem Res 51(48):15582-15592, 2012.

17. Olsson, L., Sjövall, H., and Blint, R.J., "A Kinetic Model for Ammonia Selective Catalytic Reduction over Cu-ZSM-5," Appl Catal B Environ 81(3):203-217, 2008.

18. Chatterjee, A., "An Introduction to the Proper Orthogonal Decomposition," Curr Sci. 78(7):808-817, 2000.

19. Holmes, P., Lumley, J.L., and Berkooz, G., "Turbulence, Coherent Structures, Dynamical Systems and Symmetry," (Cambridge University Press, 1998).

20. Kerschen, G., Golinval, J., Vakakis, A.F., and Bergman, L.A., "The Method of Proper Orthogonal Decomposition for Dynamical Characterization and Order Reduction of Mechanical Systems: An Overview," Nonlinear Dyn. 41(1):147-169, 2005, doi:10.1007/s11071-005-2803-2.

21. Fletcher, C.A.J., "Weighted Residual Methods," in: "Computational Techniques for Fluid Dynamics 1," (Springer, 1998), 98-162.
22. Astrid, P., "Reduction of Process Simulation Models: A Proper Orthogonal Decomposition Approach," Ph.D. thesis, Technische Universiteit Eindhoven, 2004.

23. Jeon, J., Lee, J.T., and Park, S., "Nitrogen Compounds (NO, $\mathrm{NO} 2, \mathrm{~N} 2 \mathrm{O}$, and $\mathrm{NH} 3$ ) in NOx Emissions from Commercial EURO VI Type Heavy-Duty Diesel Engines with a UreaSelective Catalytic Reduction System," Energy \& Fuels 30(8):6828-6834, 2016, doi:10.1021/acs.energyfuels.6b01331.

24. Atwell, J.A. and King, B.B., "Proper Orthogonal Decomposition for Reduced Basis Feedback Controllers for Parabolic Equations," Math Comput Model 33(1-3):119, 2001.

25. Akhtar, I., Naqvi, M., Borggaard, J., and Burns, J.A., "Using Dominant Modes for Optimal Feedback Control of Aerodynamic Forces," Proc Inst Mech Eng Part G J Aerosp Eng 227(12):1859-1869, 2013.

26. Akhiezer, N.I. and Glazman, I.M., "Theory of Linear Operators in Hilbert Space," (Courier Corporation, 2013).

27. Devarakonda, M., Parker, G., Johnson, J.H., Strots, V. et al., "Model-Based Estimation and Control System Development in a Urea-SCR Aftertreatment System," SAE Int J Fuels Lubr. 1(1):646-661, 2008, doi:10.4271/2008-01-1324.

\section{Contact Information}

\author{
Seun Olowojebutu \\ Department of Aeronautical and Automotive Engineering \\ Loughborough University \\ Loughborough, UK \\ LE11 3TT \\ ttoao@lboro.ac.uk
}

\section{Acknowledgments}

The authors would like to acknowledge the support of the Engineering and Physical Sciences Research Council (EPSRC), UK and Eminox Ltd. for the funding for this research.

\section{Definitions/Abbreviations}

$\dot{m}$ - mass flow rate $\mathrm{g} / \mathrm{s}$

$\boldsymbol{A}_{\boldsymbol{i}}$ - pre-exponential factor in rate equations

$C_{g j}$ - gas phase concentration of $\mathrm{j}^{\text {th }}$ specie, $\mathrm{mol} / \mathrm{m}^{3}$

$\boldsymbol{c}_{\boldsymbol{m}}$ - molar concentration of exhaust gas stream inside the filter wall $\mathrm{mol} / \mathrm{m}^{3}$

$C_{p g}$ - specific heat capacity of gas phase at constant pressure $\mathrm{J} / \mathrm{kg} / \mathrm{K}$

CPSI - Cell density cell/in ${ }^{2}$

$\boldsymbol{d}$ - channel width $\mathrm{m}$

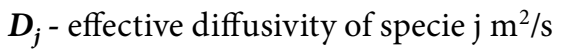

$\boldsymbol{E}_{a, j}$ - activation energy in rate equations $\mathrm{J} / \mathrm{mol}$

$\boldsymbol{H}_{\text {conv }}$ - convection of heat due to flow in channel $\mathrm{W} / \mathrm{m}^{3}$

$\boldsymbol{H}_{\boldsymbol{i}}$ - heat of formation of species i (negative for exothermic reaction) $\mathrm{J} / \mathrm{mol}$ 
$\boldsymbol{h}_{\boldsymbol{i}}$ - heat transfer coefficient $\mathrm{W} / \mathrm{m}^{2} / \mathrm{K}$

$\boldsymbol{H}_{\text {react }}$ - reaction exotherm, $\mathrm{W} / \mathrm{m}^{3}$

$\boldsymbol{H}_{\text {wall }}$ - convection of heat due to flow through wall $\mathrm{W} / \mathrm{m}^{3}$

$\boldsymbol{k}_{\boldsymbol{i}}$ - reaction rate constant

$\boldsymbol{k} \boldsymbol{i}_{\boldsymbol{m}, \boldsymbol{j}}$ - mass transfer coefficient for species j in i channel $\mathrm{m} / \mathrm{s}$

$\boldsymbol{k}_{\boldsymbol{s}}$ - wall permeability $\mathrm{m}^{2}$

$\boldsymbol{L}$ - catalyst length $\mathrm{m}$

$\boldsymbol{M W}$ - molecular weight $\mathrm{kg} / \mathrm{kmol}$

$\boldsymbol{P}$ - System pressure, $\mathrm{Pa}$

$\boldsymbol{R}$ - universal gas constant $\mathrm{J} / \mathrm{kgmolK}$

$\boldsymbol{r}_{\boldsymbol{j}} \boldsymbol{R}_{\boldsymbol{j}}$ - reaction rate for reaction $\mathrm{j} \mathrm{mol} / \mathrm{m}^{3} . \mathrm{s}$

$S_{F}$ - specific surface area of catalyst $\mathrm{m}^{-1}$

$\boldsymbol{s}_{\boldsymbol{k} i}$ - stoichiometric coefficient of surface specie $\mathrm{k}$ in reaction $\mathrm{i}$

$S \boldsymbol{V}$ - space velocity $1 / \mathrm{h}$

$\boldsymbol{T}$ - Temperature K

$\boldsymbol{t}$ - time s

$\boldsymbol{U}$ - gas flow velocity in the inlet channel $\mathrm{m} / \mathrm{s}$

$\boldsymbol{v}_{w}$ - wall velocity $\mathrm{m} / \mathrm{s}$

$\boldsymbol{w}$ - washcoat layer thickness $\mathrm{m}$

$\boldsymbol{x}$ - dimension perpendicular to wall surface m

$\boldsymbol{Y}_{\boldsymbol{j}}$ - mole fraction of specie $\mathrm{j}$

$z$-dimension perpendicular to the wall surface $m$

\section{Greek Symbols}

$\boldsymbol{\delta} \boldsymbol{z}$ - thickness of element of wall layer $\mathrm{m}$

$\boldsymbol{\rho}_{g}$ - density of gas $\mathrm{kg} / \mathrm{m} 3$

$\boldsymbol{\rho}_{\boldsymbol{s}}$ - solid density of monolith $\mathrm{kg} / \mathrm{m} 3$

$\boldsymbol{\rho}_{\boldsymbol{w}}$ - wall density $\mathrm{kg} / \mathrm{m} 3$

$\sigma_{i}$ - POD modes singular value

$\boldsymbol{\varepsilon}_{\boldsymbol{i}}$ - energy captured by i-th POD mode

$\Delta z$ - unit of control volume in axial direction

$\boldsymbol{\mu}$ - exhaust gas viscosity $\mathrm{kg} / \mathrm{ms}$

$\boldsymbol{\Phi}$ - POD orthonormal matrix

$\boldsymbol{\Omega}$ - ammonia adsorption capacity, moles of $\mathrm{NH}_{3} / \mathrm{m}^{3}$

$\boldsymbol{\varepsilon}$ - POD energy contained in data

$\boldsymbol{\theta}$ - ammonia surface coverage

$\lambda$ - eigenvalues
Subscript and Superscript

1 s, 2 s - channel-solid interface

g - exhaust gas

i - channel index, 1 = inlet, 2 = outlet

in - inlet channel, inlet conditions

j - specie index, reaction index

$\mathbf{k}$ - species index

out - outlet channel

s - solid

w - substrate wall

\section{Abbreviations}

1D - one dimensional

AMOX - ammonia oxidation catalyst

ANR - ammonia- $\mathrm{NO}_{\mathrm{x}}$ ratio

ASC - ammonia surface coverage

deNOx - $\mathrm{NO}_{\mathrm{x}}$ conversion

DPF - diesel particulate filter

EATS - exhaust after-treatment systems

ECU - engine control unit

LQR - linear quadratic regulator

MOR - model order reduction

NOx - nitrogen oxides

ODE - ordinary differential equation

OL - open loop

PDE - partial differential equation

PM - particulate matter / soot

POD - proper orthogonal decomposition

ROM - reduced order model

SCR - selective catalytic reduction

SCR-in-DPF - selective catalytic reduction catalyst integrated on diesel particulate filter

WHTC - world harmonised test cycle

All rights reserved. No part of this publication may be reproduced, stored in a retrieval system, or transmitted, in any form or by any means, electronic, mechanical, photocopying, recording, or otherwise, without the prior written permission of the copyright holder. 